\title{
Теория графов
}

(C)Смирнов А. В., 2018

DOI: $10.18255 / 1818-1015-2018-4-388-401$

УДК 519.17

\section{Остовное дерево в делимом кратном графе}

\author{
Смирнов А.В. \\ получена 29 июля 2018
}

Аннотация. В статье рассматриваются неориентированные кратные графы произвольной натуральной кратности $k>1$. Кратный граф содержит ребра трех типов: обычные, кратные и мультиребра. Ребра последних двух типов представляют собой объединение $k$ связанных ребер, которые соединяют 2 или $k+1$ вершину соответственно. Связанные ребра могут использоваться только согласованно. Если вершина инцидентна кратному ребру, то она может быть инцидентна другим кратным ребрам, а также она может быть общим концом $k$ связанных ребер мультиребра. Если вершина является общим концом мультиребра, то она не может быть общим концом никакого другого мультиребра.

Особое внимание уделяется классу делимых кратных графов, которые отличаются возможностью выделения $k$ частей, согласованных на всех связанных ребрах и не содержащих общих ребер. Каждая из частей является обычным графом.

Вводится понятие кратного дерева, определяюся его основные свойства. В отличие от обычных деревьев количество ребер в кратных деревьях не фиксировано. Для делимых деревьев в работе приводится и обосновывается оценка минимального и максимального количества ребер.

Далее определяются понятия остовного дерева и полного остовного дерева. Для делимых графов доказывается критерий полноты остовного дерева. Также доказано, что полное остовное дерево всегда существует в делимом графе.

Если кратный граф является взвешенным, то для него можно поставить задачу о минимальном остовном дереве, а также о минимальном полном остовном дереве. В работе предложен эвристический алгоритм поиска минимального полного остовного дерева в делимом графе.

Ключевые слова: кратный граф, кратное дерево, делимый граф, остовное дерево, полное остовное дерево, минимальное остовное дерево

Для цитирования: Смирнов А. В., "Остовное дерево в делимом кратном графе", Моделирование и анализ информационных систем, 25:4 (2018), 388-401.

\section{Об авторах:}

Смирнов Александр Валерьевич, orcid.org/0000-0002-0980-2507, канд. физ.-мат. наук, доцент,

Ярославский государственный университет им. П.Г. Демидова,

ул. Советская, 14, г. Ярославль, 150003 Россия, e-mail: alexander_sm@mail.ru

Благодарности:

Работа выполнена при поддержке Российского фонда фундаментальных исследований (проект № 17-07-00823 А).

\section{Введение}

В данной статье будет дано понятие кратного дерева и рассмотрена задача о минимальном остовном дереве в делимом кратном графе. Определение кратного графа 
кратности $k>1$ и делимого кратного графа было сформулировано в статье [1]. Там же была рассмотрена задача о кратчайшем кратном пути между двумя вершинами. Кратные графы содержат три типа ребер (обычные, кратные и мультиребра) и являются обобщением обычных графов - по сути, обычный граф имеет кратность $k=1$.

Среди других известных обобщений графов наиболее близкими нам концепциями являются мультиграфы, гиперграфы (см., например, [2] - [3]), а также метаграфы (см. [4] - [5]). Действительно, как и в мультиграфах, в кратных графах допускается наличие нескольких ребер между парой вершин (набор таких ребер мы будем в дальнейшем называть кратным ребром), однако в случае кратного графа количество таких ребер должно быть строго равным $k$. В кратных графах присутствуют мультиребра, соединяющие между собой $k+1$ вершину. Но в отличие от гиперребер гиперграфа, мультиребро представляется в виде $k$ связанных ребер, имеющих один общий конец, причем все эти $k$ ребер должны использоваться согласованно. По сути, понятие мультиребра близко понятию ребра между вершиной и метавершиной в метаграфе. При этом в метаграфе, напомним, метапуть между двумя метавершинами фактически моделирует причинно-следственные связи в некоторой предметной области. Однако в кратном графе используется принципиально иной подход к определению пути: кратный путь должен состоять ровно из $k$ обычных путей, проходящих по обычным ребрам, а также по связанным ребрам кратных и мультиребер; при этом пути должны быть согласованы (одинаковы) на кратных и мультиребрах. Поэтому кратный граф нельзя считать частным случаем метаграфа.

Отметим также, что частным случаем кратного графа является кратная сеть (см. [6] - [7]). Задача о наибольшем потоке в кратной сети обобщает классическую задачу (см. [8]) и имеет ряд приложений в сфере экономики, управления, финансов. В частности, кратные сети и потоки используются для поиска решения $N P$-полной задачи целочисленного сбалансирования трех- и четырехмерной матрицы (см., например, [9] - [10]).

\section{1. Кратный граф и кратный путь}

Прежде чем ввести определение кратного дерева, напомним несколько понятий, относящихся к кратным графам (подробней см. в [1]).

Определение 1. В качестве кратного графа произвольной натуральной кратности $k>1$ рассматривается граф $G(X, E)$, между вериинами которого могут быть ребра одного из 3 видов:

1) обычное ребро $e^{o}$; множество обычных ребер обозначим через $E^{o}$;

2) кратное ребро $e^{k}$ между двумя вершинами, которое состоит из $k$ одинаковых связанных ребер; связанные ребра кратного ребра могут использоваться только согласованно; множество кратных ребер обозначим через $E^{k}$;

3) связанное ребро е между двумя вершинами, имеющее один общий конец с другими $k-1$ ребрами (у любых двух из $k$ связанных ребер только один конеи, является общим); множество связанных общей вершиной ребер будем называть 
мультиребром $e^{m}$; связанные ребра мультиребра могут использоваться только согласованно; множество мультиребер обозначим через $E^{m}$.

Если вершина иниидентна какому-либо кратному ребру, то она может быть инцидентна другим кратным ребрам, а также она может быть общим кониом какого-либо мультиребра.

Если вершина является общим концом какого-либо мультиребра, то она не может быть общим кониом никакого другого мультиребра.

Мы ограничимся рассмотрением неориентированных кратных графов.

Определение 2. Делимым кратным графом назовем такой граф, в котором между двумя кониами одного мультиребра не существует пути, проходящего только по обычным ребрам.

Очевидно, что при удалении всех мультиребер делимый граф распадется на $n$ компонент связности (связность здесь понимается в том же смысле, что и для обычных графов), каждая из которых содержит только кратные ребра либо только обычные ребра. При этом связанные ребра каждого мультиребра можно пронумеровать от 1 до $k$ таким образом, что каждой компоненте связности, содержащей только обычные ребра, будут инцидентны связанные ребра мультиребер с одинаковыми номерами.

Определение 3. Частью $G_{i}(i \in \overline{1, k})$ делимого графа $G(X, E)$ назовем подграф, содержащий связанные ребра с номером $i$ всех кратных и мультиребер, а также компоненты связности, состоящие из обычных ребер и иниидентные $i$-м связанным ребрам всех мультиребер.

Очевидно, что каждая часть $G_{i}$ является обычным графом. При этом возможность выделения частей $G_{i}$ является особенностью делимых графов. В общем случае получить части $G_{i}$ не удастся.

Определение 4. Вериина является висячей, если она иниидентна только одному ребру.

Дадим теперь определение кратного пути. Основное отличие кратного пути от пути в обычном графе состоит в том, что связанные ребра каждого кратного и мультиребра должны проходиться в этом пути согласованно.

Определение 5. $S(x, y)=\cup_{i=1}^{k} S^{i}(x, y)$ является кратным путем из вершины $x$ в вершину у в графе $G(X, E)$, если выполнены следующие условия:

1) $S^{i}(x, y)=\left(\left\{x, v_{1}^{i}\right\},\left\{v_{1}^{i}, v_{2}^{i}\right\}, \ldots,\left\{v_{p-1}^{i}, v_{p}^{i}\right\},\left\{v_{p}^{i}, y\right\}\right)$, где $p \geq 0$, - последовательность ребер, представляющая собой обычный (некратный) путь из х в $y$, где каждое ребро $\{a, b\}$ является либо обычным ребром в графе $G(X, E)$, либо $i$-м связанным ребром кратного или мультиребра. Если в путь $S(x, y)$ не входит ни одного кратного или мультребра, то $S^{2}(x, y)=S^{3}(x, y)=\ldots=S^{k}(x, y)=\varnothing$;

2) вершины, не являюшиеся общим кониом связанных ребер мультиребра и не иниидентные кратным ребрам, могут встречаться в $S^{i}(x, y)$ несколько раз, то есть $S^{i}(x, y)$ может содержать иикль;

3) вершины, инцидентные кратным ребрам либо являющиеся общим концом мультиребра, не могут встретиться в $S^{i}(x, y)$ дважды; 
4) любое обычное ребро может встречаться в $S^{i}(x, y)$ несколько раз, причем направления, в которых оно проходится в разных вхождениях, могут не совпадаmъ;

5) обычное ребро, входящее в $S^{i}(x, y)$, может также входить в любой $S^{j}(x, y)$, $j \neq i$;

6) все пути $S^{i}(x, y)$ согласованы (одинаковы) на общей части. Это условие означает, что если связанное ребро какого-то кратного или мультиребра входит в некоторый путь $S^{i}(x, y)$, то остальные связанные ребра должны входить во все $S^{j}(x, y), j \neq i$ (по одному связанному ребру в каждый $S^{j}(x, y)$ ). При этом порядок вхождения всех кратных и мультиребер во все $S^{i}(x, y)$ одинаков;

7) если $S(x, y)$ содержит мультиребро $\left\{x_{0},\left\{x_{1}, \ldots, x_{k}\right\}\right\}$, проходимое в направлении от общего кониа, то он не может содержать никакого другого мультиребра $\left\{y_{0},\left\{x_{1}, \ldots, x_{k}\right\}\right\}$, проходимого в том же направлении. Аналогичное условие должно выполняться и в случае движения к общему кониу.

Определение 6. Кратный путь $S(x, y)$ является кратным циклом, если $x=y u$ $S(x, y) \neq \varnothing$.

Теперь мы можем ввести понятие связности для кратного графа.

Определение 7. Кратный граф $G(X, E)$ является связным, если одновременно выполнены два условия:

1) для любых двух вершин $x \in X, y \in X$, каждая из которых либо иниидентна кратному ребру, либо является общим концом мультиребра, существует кратныи nymв $S(x, y)$;

2) невозможно выделить такой подграф $G^{\prime} \subset G$, который будет содержать только обычные ребра, и при этом подграфы $G^{\prime} u G \backslash G^{\prime}$ не будут соединены ни одним ребром (обычным ребром или связанным ребром мультиребра).

В отличие от обычных графов связность кратного графа не предполагает наличие кратных путей из каждой вершины в каждую. Фактически в связном кратном графе между каждой парой вершин должен существовать обычный (некратный) путь, использующий связанные ребра кратных и мультиребер несогласованно, а кратные пути обязательно должны существовать только для пар вершин, каждая из которых инцидентна кратным ребрам или является общим концом мультиребра.

Отметим, что для делимого кратного графа определение связности может быть переписано в более простой форме, что обусловлено структурой графа.

Определение 8. Делимый кратный граф $G(X, E)$ является связным, если одновременно выполнены два условия:

1) для любых двух вершин $x \in X, y \in X$, каждая из которых либо иниидентна кратному ребру, либо является общим концом мультиребра, существует кратныи nyms $S(x, y)$;

2) каждая из частей $G_{i}$ является связным графом.

\section{2. Кратные деревья и их свойства}

Определение 9. Кратное дерево - это связный кратный граф без циклов. 
Пример 1.

Рассмотрим простые примеры кратных деревьев кратности 2 с похожей структурой (жирными линиями отмечены кратные ребра, раздваивающимися - мультиребра).
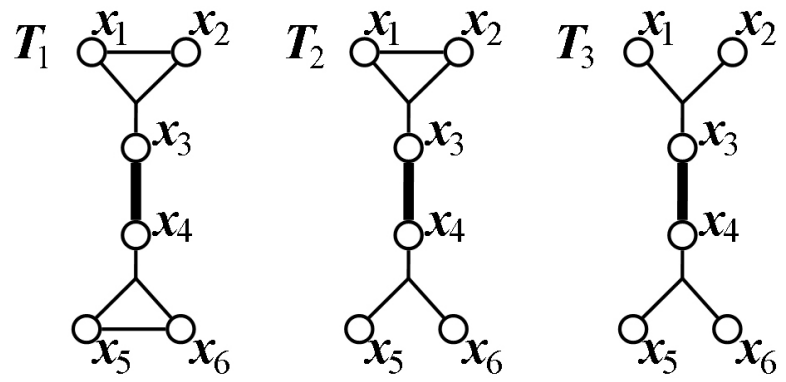

Рис. 1. Примеры кратных деревьев

Fig. 1. Examples of multiple trees

Все три представленых на рис. 1 графа являются деревьями: действительно, для каждого из них выполнено условие связности (в кратном смысле), и ни в одном из графов невозможно построить кратный цикл. При этом дерево $T_{3}$ является делимым, а деревья $T_{1}$ и $T_{2}-$ нет.

Хотя количество вершин во всех деревьях одинаково, количество ребер различно - это особенность кратных деревьев. Оценка минимального и максимального количества ребер в делимом дереве будет приведена и обоснована ниже.

Особо отметим, что в кратном дереве может не быть ни одной висячей вершины (пример - дерево $T_{1}$ ).

Определение 10. Два кратных пути $S_{1}(x, y)$ u $S_{2}(x, y)$ назовем подобными, если для них выполнены следующие условия:

1) порядок вхождения кратных и мультиребер в пути $S_{1}(x, y)$ u $S_{2}(x, y)$ одинаков;

2) путь $S_{2}(x, y)$ может быть получен перестановкой некратных участков пути $S_{1}(x, y)$ между простыми путями $S_{1}^{i}(x, y)$, причем менять местами можно только участки, заключенные между общими концами двух мультиребер либо между начальной/конечной вершиной пути и общим концом какого-либо мультиребра.

Пример 2.

Чтобы проиллюстрировать определение подобных путей, рассмотрим дерево $T_{1}$ из примера 1 (рис. 1). В этом дереве существует два различных кратных пути из $x_{1}$ в $x_{5}$. Первый путь $S_{1}\left(x_{1}, x_{5}\right)$ состоит из двух обычных путей

$$
\begin{gathered}
S_{1}^{1}\left(x_{1}, x_{5}\right)=\left(\left\{x_{1}, x_{3}\right\},\left\{x_{3}, x_{4}\right\}, \underline{\left\{x_{4}, x_{5}\right\}}\right), \\
S_{1}^{2}\left(x_{1}, x_{5}\right)=\left(\left\{x_{1}, x_{2}\right\},\left\{x_{2}, x_{3}\right\},\left\{x_{3}, x_{4}\right\}, \underline{\underline{\left\{x_{4}, x_{6}\right\}}}, \underline{\underline{\left\{x_{6}, x_{5}\right\}}}\right),
\end{gathered}
$$

а второй путь $S_{2}\left(x_{1}, x_{5}\right)$ состоит из двух обычных путей

$$
S_{2}^{1}\left(x_{1}, x_{5}\right)=\left(\left\{x_{1}, x_{3}\right\},\left\{x_{3}, x_{4}\right\}, \underline{\underline{\left\{x_{4}, x_{6}\right.}}, \underline{\underline{\left\{x_{6}, x_{5}\right\}}}\right),
$$




$$
S_{2}^{2}\left(x_{1}, x_{5}\right)=\left(\left\{x_{1}, x_{2}\right\},\left\{x_{2}, x_{3}\right\},\left\{x_{3}, x_{4}\right\}, \underline{\left\{x_{4}, x_{5}\right\}}\right)
$$

Нетрудно заметить, что путь $S_{2}\left(x_{1}, x_{5}\right)$ получается из пути $S_{1}\left(x_{1}, x_{5}\right)$ перестановкой некратных участков пути между простыми путями (участки, отмеченные одинарным и двойным подчеркиванием). При этом переставляемые участки заключены между общим концом мультиребра и конечной вершиной пути. Следовательно, пути $S_{1}\left(x_{1}, x_{5}\right)$ и $S_{2}\left(x_{1}, x_{5}\right)$ подобны.

Справедливо следующее утверждение.

Теорема 1. Связный кратный граф $G(X, E)$ является деревом тогда и только тогда, когда для любой пары вершин $x \in X u y \in X$ выполнено одно из двух условий $(P(x, y)$ - множество всех кратных путей из $x$ в $y)$ :

1) $P(x, y)=\varnothing$; $i \neq j$.

2) $P(x, y)=\left\{S_{1}(x, y), \ldots, S_{p}(x, y)\right\}$, при этом $S_{i}(x, y)$ подобен $S_{j}(x, y)$ для всех

\section{Доказательство.}

Необходимость. Пусть $G(X, E)$ - кратное дерево. Предположим противное: в графе $G(X, E)$ существуют две различные вершины $x$ и $y$ такие, что между ними можно построить кратные пути $S_{1}(x, y)$ и $S_{2}(x, y)$, не являющиеся подобными.

Это означает, что в путях $S_{1}(x, y), S_{2}(x, y)$ обязательно найдутся непустые участки $S_{1}^{\prime}\left(x_{0}, y_{0}\right) \subseteq S_{1}(x, y)$ и $S_{2}^{\prime}\left(x_{0}, y_{0}\right) \subseteq S_{2}(x, y)$, не содержащие одинаковых ребер. Эти участки могут быть как кратными (содержать кратные и/или мультиребра), так и некратными (содержать только обычные ребра). $x_{o}$ может совпадать с $x, y_{0}-$ с $y$.

В любом случае $S_{1}^{\prime}\left(x_{0}, y_{0}\right), S_{2}^{\prime}\left(x_{0}, y_{0}\right)$ представляют собой кратные пути из $x_{0}$ в $y_{0}$. Граф неориентированный, поэтому, обратив $S_{2}^{\prime}\left(x_{0}, y_{0}\right)$, мы получим путь $S_{2}^{\prime}\left(y_{0}, x_{0}\right)$. Теперь у нас есть путь из $x_{0}$ в $y_{0}$ и путь из $y_{0}$ в $x_{0}$, не содержащие общих ребер. Объединение $S_{1}^{\prime}\left(x_{0}, y_{0}\right)$ и $S_{2}^{\prime}\left(y_{0}, x_{0}\right)$ дает кратный цикл, что приводит к противоречию.

Достаточность. Пусть для всех пар вершин выполнено условие теоремы. Предположим противное: граф $G(X, E)$ не является кратным деревом.

Это означает, что в графе $G(X, E)$ существует кратный цикл $C$, содержащий не менее трех вершин. Пусть вершины $x_{0} \in C, y_{0} \in C$. Тогда кратный цикл $C$ можно представить в виде

$$
C=S_{1}\left(x_{0}, y_{0}\right) \cup S_{2}\left(y_{0}, x_{0}\right),
$$

где $S_{1}, S_{2}$ - кратные пути. При этом очевидно, что найдется ребро $e$ такое, что $e \in S_{1}\left(x_{0}, y_{0}\right)$ и $e \notin S_{2}\left(y_{0}, x_{0}\right)$ (иначе $C=\varnothing$ ). Граф неориентированный, поэтому, обратив $S_{2}\left(y_{0}, x_{0}\right)$, мы получим путь $S_{2}\left(x_{0}, y_{0}\right)$. Поскольку $e \notin S_{2}\left(y_{0}, x_{0}\right)$, то $e \notin$ $S_{2}\left(x_{0}, y_{0}\right)$. Следовательно, пути $S_{1}\left(x_{0}, y_{0}\right)$ и $S_{2}\left(x_{0}, y_{0}\right)$ не являются подобными, что приводит к противоречию.

Теорема доказана.

Следствие 1. Связный делимый граф является деревом тогда и только тогда, когда для любой пары вершин $x \in X u y \in X$ существует не более одного пути $S(x, y)$. 
Отметим, что в любом кратном дереве, как и в обычном, заведомо есть $|X|-1$ обычное или связанное ребро. Однако количество ребер не фиксировано и может быть больше.

Пусть $T(X, E)$ - делимое кратное дерево кратности $k$. Обозначим через $n_{i}$ количество вершин в частях $T_{i}(i \in \overline{1, k})$ этого дерева. Зная количество мультиребер в дереве, мы сможем оценить общее количество обычных и связанных ребер в нем.

Теорема 2. В кратном делимом дереве количество обычных и связанных ребер $c=\left|E^{o}\right|+k\left|E^{k}\right|+k\left|E^{m}\right|$ оценивается по формуле

$$
\sum_{i=1}^{k} n_{i}-k \leq c \leq \sum_{i=1}^{k} n_{i}-k+k\left(\left|E^{m}\right|-1\right)
$$

при условии, что $\left|E^{m}\right| \geq 1$.

Доказательство. Сначала докажем, что

$$
\sum_{i=1}^{k} n_{i}-k \leq c
$$

Делимое кратное дерево является связным кратным графом, следовательно, каждая его часть $T_{i}(i \in \overline{1, k})$ - связный обычный граф. Значит, каждая из частей $G_{i}$ содержит не менее $n_{i}-1$ ребер, откуда следует требуемое соотношение.

Докажем теперь, что

$$
c \leq \sum_{i=1}^{k} n_{i}-k+k\left(\left|E^{m}\right|-1\right)
$$

Пусть $n_{0}^{\prime}$ - количество вершин, инцидентных кратным ребрам или являющихся общим концом мультиребер. В дереве $T(X, E)$ между ними может быть не более чем $n_{0}^{\prime}-1$ кратное ребро $\left(k\left(n_{0}^{\prime}-1\right)\right.$ связанных ребер). В противном случае мы получим цикл по кратным ребрам. Пусть $n_{i}^{\prime}-$ количество вершин в части $T_{i}(i \in \overline{1, k})$, инцидентных обычным ребрам или являющихся отдельными концами мультиребер. Между ними может быть не более чем $n_{i}^{\prime}-1$ обычное ребро. В противном случае мы получим цикл по обычным ребрам. Заметим, что $n_{0}^{\prime}+n_{i}^{\prime}=n_{i}$. Тогда

$$
c \leq k\left(n_{0}^{\prime}-1\right)+k\left|E^{m}\right|+\sum_{i=1}^{k}\left(n_{i}^{\prime}-1\right)=\sum_{i=1}^{k} n_{i}-k+k\left(\left|E^{m}\right|-1\right),
$$

что и требовалось доказать.

Теорема доказана.

Отметим, что случай $\left|E^{m}\right|=0$, не рассмотренный в теореме, является вырожденным - кратное дерево, не содержащее мультиребер, по сути, эквивалентно обычному дереву.

Также отметим, что при $\left|E^{m}\right|=1$ оценка для $с$ обращается в равенство. 


\section{3. Остовные деревья в кратном графе. Критерий полноты остовного дерева в делимом графе}

Пусть $G(X, E)$ - связный кратный граф.

Определение 11. Остовным деревом в кратном графе $G(X, E)$ называется кратное дерево $T\left(X, E^{\prime}\right)$, для которого $E^{\prime} \subseteq E$.

Заметим, что в остовном дереве заведомо будут существовать кратные пути $S(x, y)$ для всех вершин $x, y$, инцидентных кратным ребрам или являющихся общим концом мультиребер. Однако, если хотя бы одна из вершин $x, y$ инцидентна обычному ребру или отдельному связанному ребру мультиребра, существование пути $S(x, y)$ не гарантировано даже в том случае, когда такой путь существует в исходном графе $G(X, E)$.

Определение 12. Остовное дерево $T\left(X, E^{\prime}\right)$ в кратном графе $G(X, E)$ является полным, если для любой пары вершин $x \in X, y \in X$ кратный путь $S_{T}(x, y)$ в дереве $T\left(X, E^{\prime}\right)$ существует тогда и только тогда, когда существует кратныи nуть $S_{G}(x, y)$ в исходном графе $G(X, E)$.

Определение 13. Множеством достижимости по обычным ребрам для некоторой вершины $x$ назовем множество $R_{x}^{o}$ всех вершин у таких, что существует путь из х в $y$, проходящий только по обычным ребрам.

Множество достижимости по обычным ребрам определяется только для вершин, не инцидентных кратным ребрам и не являющихся общим концом мультиребра.

Теорема 3. Пусть $T\left(X, E^{\prime}\right)$ - остовное дерево в делимом кратном графе $G(X, E)$. Остовное дерево $T\left(X, E^{\prime}\right)$ является полным тогда и только тогда, когда на каждом множестве достижимости по обычным ребрам $R_{x}^{o}$ графа $G(X, E)$ построено обичное дерево $T^{o}\left(R_{x}^{o}, E_{x}^{o}\right)$, где $E_{x}^{o} \subseteq E^{\prime}, E_{x}^{o} \subseteq E^{o}$.

Доказательство. По теореме 3 статьи [1] (критерий существования кратного пути между двумя вершинами), учитывая связность графа $G(X, E)$, получаем, что в графе $G(X, E)$ могут существовать кратные пути $S_{G}(x, y)$ одного из двух видов:

1) вершины $x$ и $y$ инцидентны кратным ребрам или являются общим концом мультиребер;

2 ) вершины $x$ и $y$ инцидентны обычным ребрам, и путь $S_{G}(x, y)$ проходит только по обычным ребрам, то есть $y \in R_{x}^{o}$.

Отметим, что если для пары вершин $x, y$ в графе $G(X, E)$ существует путь $S_{G}(x, y)$ первого типа, то существование пути $S_{T}(x, y)$ в остовном дереве $T\left(X, E^{\prime}\right)$ обеспечивается его связностью и не зависит от полноты дерева.

Пути второго типа существуют в исходном графе тогда и только тогда, когда вершина $y \in R_{x}^{o}$.

Поэтому для полноты дерева $T\left(X, E^{\prime}\right)$ необходимо и достаточно, чтобы в этом дереве вершины каждого множества достижимости $R_{x}^{o}$ исходного графа могли быть связаны путями, проходящими только по обычным ребрам. Это возможно только в том случае, когда на множестве $R_{x}^{o}$ построен связный обычный граф $T^{o}\left(R_{x}^{o}, E_{x}^{o}\right)$, 
где $E_{x}^{o} \subseteq E^{\prime}$ и $E_{x}^{o} \subseteq E^{o}$. При этом $T^{o}\left(R_{x}^{o}, E_{x}^{o}\right)$ обязательно является деревом (иначе возникнут циклы по обычным ребрам).

Теорема доказана.

Отметим, что условие теоремы справедливо только для делимых графов. Для произвольного кратного графа теорема 3 не будет верна. Рассмотрим соответствующие примеры.

Пример 3.

Пусть имеется кратный граф $G$ кратности 2 , изображенный на рис. 2.
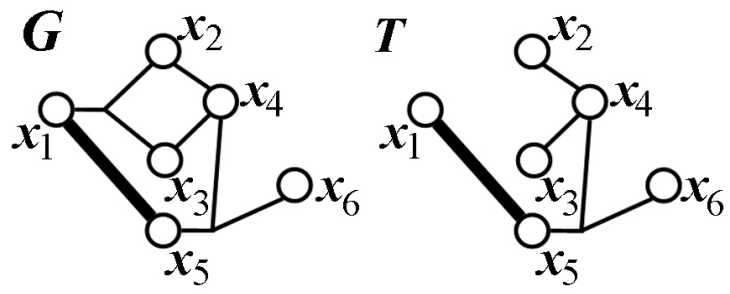

Рис. 2. Остовное дерево в кратном графе

Fig. 2. Spanning tree in a multiple graph

Заметим, что этот граф не является делимым, поскольку существует путь из вершины $x_{2}$ в вершину $x_{3}$, проходящий только по обычным ребрам, и при этом данные вершины являются концами одного мультиребра.

Нетрудно убедиться, что граф $T$ является остовным деревом для $G$. Поскольку в графе $G$ существует путь $S\left(x_{1}, x_{5}\right)$, а в графе $T$ - нет, остовное дерево $T$ не является полным. Однако условие теоремы 3 выполнено: в исходном графе $G$ два множества достижимости по обычным ребрам - $R_{x_{2}}^{o}=\left\{x_{2}, x_{3}, x_{4}\right\}$ и $R_{x_{6}}=\left\{x_{6}\right\}$; на каждом из этих множеств в графе $T$ построено обычное дерево. Таким образом, для произвольного кратного графа построение обычных деревьев на множествах достижимости $R_{x}^{o}$ не является достаточным для полноты остовного дерева.

Отметим также, что в данном примере единственно возможное полное остовное дерево будет совпадать с графом $G$.

Пример 4.

Пусть теперь имеется кратный граф $G$, показанный на рис. 3.

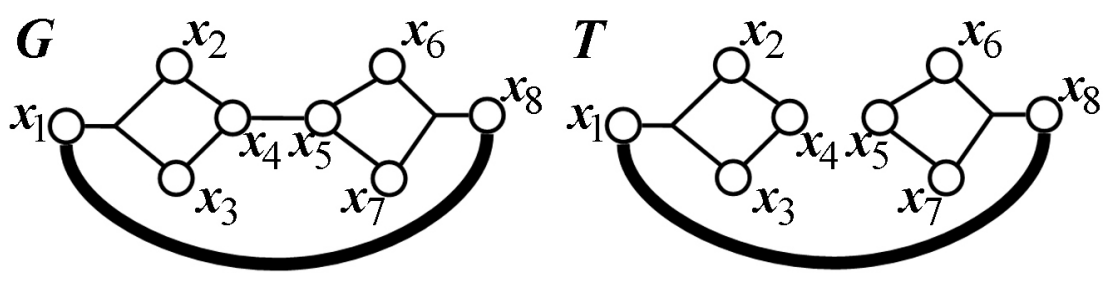

Рис. 3. Полное остовное дерево в кратном графе

Fig. 3. Complete spanning tree in a multiple graph

Граф $G$ не является делимым, кроме того, в нем имеются кратные пути между каждой парой вершин. 
Удаляя обычное ребро $\left\{x_{4}, x_{5}\right\}$, мы получим полное остовное дерево T. При этом в исходном графе было единственное множество достижимости по обычным ребрам $R_{x_{2}}=\left\{x_{2}, x_{3}, x_{4}, x_{5}, x_{6}, x_{7}\right\}$, на котором в остовном дереве $T$ построен несвязный обычный граф. Следовательно, для произвольного кратного графа построение обычных деревьев на множествах достижимости $R_{x}^{o}$ не является необходимым для полноты остовного дерева.

Отметим, что если бы мы в исходном графе $G$ вместо обычного ребра $\left\{x_{4}, x_{5}\right\}$ удалили кратное ребро $\left\{x_{1}, x_{8}\right\}$ или любое из мультиребер, то мы все равно бы получили полное остовное дерево (при этом на множестве $R_{x_{2}}$ было бы построено обычное дерево). Любое другое остовное дерево в данном графе не будет полным.

Независимо от того, делимый граф или нет, проверка полноты остовного дерева выполняется за полиномиальное число шагов.

Действительно, поиск множеств достижимости в кратном графе полиномиален (алгоритм 2 статьи [1]). Для делимого графа остается проверить, что в кратном дереве на каждом множестве достижимости построено обычное дерево, что может быть сделано с помощью стандартных полиномиальных алгоритмов для обычных графов.

Для произвольного кратного графа нужно проверить наличие путей между всеми возможными парами вершин. Затем для тех пар, где пути существуют, проверить наличие путей в остовном дереве. Количество пар вершин полиномиально, а каждую проверку можно осуществить с помощью полиномиальных алгоритмов из статьи [1].

\section{4. Задача о минимальном остовном дереве в кратном графе. Алгоритм для делимых графов}

Определение 14. Целочисленная функиия $l(e)$, определенная для всех ребер е $\in E$, является длиной (весом) ребра в кратном графе $G(X, E)$, если выполнено следующеe:

1) $l(e)>0$ для любого ребра $е$;

2) если е является кратным или мультиребром, то $l\left(e_{1}\right)=l\left(e_{2}\right)=\ldots=l\left(e_{k}\right) u$ $l(e)=k \cdot l\left(e_{1}\right)$, где $e_{1}, \ldots, e_{k}-$ это связанные ребра данного ребра е.

Тогда вес кратного графа $G(X, E)$ будет определяться по формуле

$$
w(G(X, E))=\sum_{e \in E} l(e)
$$

Поставим две задачи о минимальном остовном дереве.

Задача 1 (минимальное остовное дерево). B кратном графе $G(X, E)$ mребуется найти такое остовное дерево $T^{\mathrm{min}}\left(X, E^{\prime}\right)$, что для любого остовного дерева $T\left(X, E^{\prime \prime}\right)$ выполнено

$$
w\left(T^{\min }\left(X, E^{\prime}\right)\right) \leq w\left(T\left(X, E^{\prime \prime}\right)\right) .
$$


Задача 2 (минимальное полное остовное дерево). В кратном графе $G(X, E)$ требуется найти такое полное остовное дерево $T_{\text {complete }}^{\min }\left(X, E^{\prime}\right)$, что для любого полного остовного дерева $T_{\text {complete }}\left(X, E^{\prime \prime}\right)$ выполнено

$$
w\left(T_{\text {complete }}^{\min }\left(X, E^{\prime}\right)\right) \leq w\left(T_{\text {complete }}\left(X, E^{\prime \prime}\right)\right) .
$$

Больший интерес здесь представляет задача 2 ввиду того, что в полном остовном дереве есть кратные пути между всеми парами вершин, для которых есть кратные пути в исходном графе. При этом очевидно, что

$$
w\left(T^{\min }\left(X, E^{\prime}\right)\right) \leq w\left(T_{\text {complete }}^{\min }\left(X, E^{\prime \prime}\right)\right) .
$$

Существование остовного дерева в связном кратном графе очевидно, поэтому задача 1 всегда разрешима. Разрешимость задачи 2 для делимых графов устанавливается следующей теоремой.

Теорема 4. В свлзном делимом кратном графе $G(X, E)$ всегда существует полное остовное дерево $T\left(X, E^{\prime}\right)$.

Доказательство. Если в графе $G(X, E)$ нет обычных ребер $\left(E^{o}=\varnothing\right)$, то любое остовное дерево является полным (следует из определения связного кратного графа и остовного дерева).

Пусть в графе $G(X, E)$ множество $E^{o} \neq \varnothing$.

Для каждого множества $R_{x}^{o}$ исходного графа, где вершина $x$ инцидентна обычному ребру, построим обычное дерево $T_{x}^{o}\left(R_{x}^{o}, E_{x}^{o}\right)$. Здесь $E_{x}^{o} \subseteq E^{o}$. Будем считать каждое такое дерево единой квазивершиной $T_{i}(i \in \overline{1, q})$. Сформируем множество всех квазивершин $Q=\left\{T_{1}, \ldots, T_{q}\right\}$ и построим кратный граф $G_{Q}\left(X_{Q}, E_{Q}\right)$, где $X_{Q}=(X \cup Q) \backslash\left(\cup R_{x}^{o}\right), E_{Q}=E^{k} \cup E_{Q}^{m}$. Множество $E_{Q}^{m}$ получается из множества $E^{m}$ заменой каждого мультиребра $\left\{a,\left\{a_{1}, \ldots, a_{k}\right\}\right\}$ на мультиребро $\left\{a,\left\{T_{i_{1}}, \ldots, T_{i_{k}}\right\}\right\}$ таким образом, что $a_{j} \in T_{i_{j}}$ для всех $j \in \overline{1, k}$. В силу делимости исходного графа $i_{j} \neq i_{p}$, если $j \neq p$.

Следовательно, мы получили связный делимый кратный граф $G_{Q}\left(X_{Q}, E_{Q}\right)$ без обычных ребер. Значит, в нем любое остовное дерево $T_{Q}\left(X_{Q}, E_{Q}^{\prime}\right)$ является полным. Выполняя в дереве $T_{Q}$ обратную замену всех квазивершин $T_{i}$ на обычные деревья $T_{x}^{o}$, мы получим остовное дерево $T\left(X, E^{\prime}\right)$ для исходного графа $G(X, E)$. При этом для дерева $T\left(X, E^{\prime}\right)$ выполнено условие теоремы 3 , следовательно, оно является полным.

Теорема доказана.

Пусть $G(X, E)$ - делимый кратный граф. Рассмотрим следующий эвристический алгоритм для задачи 2. Несмотря на то, что в нем обобщается известный алгоритм Краскала (см. [11]), решение не всегда будет точным. Более того, можно предположить, что задачи 1 и 2 для произвольных кратных графов $N P$-полны.

\section{Алгоритм.}

1. С помощью алгоритма Краскала строим минимальные остовные деревья $T_{x}^{o}\left(R_{x}^{o}, E_{x}^{o}\right)$ для каждого множества достижимости по обычным ребрам $R_{x}^{o}$ исходного графа $G(X, E)$.

2. Будем считать каждое построенное дерево $T_{x}^{o}$ квазиверииной $T_{i}$. Сформируем множество всех квазивершин $Q=\left\{T_{1}, \ldots, T_{q}\right\}$ и построим кратный граф 
$G_{Q}\left(X_{Q}, E_{Q}\right)$, где $X_{Q}=(X \cup Q) \backslash\left(\cup R_{x}^{o}\right), E_{Q}=E^{k} \cup E_{Q}^{m}\left(E_{Q}^{m}\right.$ получается из $E^{m}$ так же, как в доказательстве теоремы 4). Этот кратный граф не содержит обычных ребер.

3. Будем искать полное остовное дерево $T_{Q}\left(X_{Q}, E_{Q}^{\prime}\right)$ в графе $G_{Q}\left(X_{Q}, E_{Q}\right)$ следующим образом.

3.1. Все ребра из $E_{Q}$ сортируем по возрастанию весов и объявляем непросмотренными. Устанавливаем $E_{Q}^{\prime}=\varnothing$.

3.2. Возьмем очередное непросмотренное ребро $e \in E_{Q}$. Если таких ребер нет, переходим на шаг 3.7.

3.3. Если $e$ - кратное ребро и в графе $T_{Q}\left(X_{Q}, E_{Q}^{\prime}\right)$ нет кратного пути из одного конца ребра $e$ в другой, то добавляем $e$ в $E_{Q}^{\prime}$ и переходим на шаг 3.5 .

3.4. Если $e$ - мультиребро вида $\left\{a,\left\{T_{i_{1}}, \ldots, T_{i_{k}}\right\}\right\}$ и в графе $T_{Q}\left(X_{Q}, E_{Q}^{\prime}\right)$ нет мультиребра $\left\{b,\left\{T_{i_{1}}, \ldots, T_{i_{k}}\right\}\right\}$ такого, что существует кратный путь $S(a, b)$, то добавляем e B $E_{Q}^{\prime}$.

3.5. Ребро е становится просмотренным.

3.6. Если $T_{Q}\left(X_{Q}, E_{Q}^{\prime}\right)$ - связный граф, переходим на шаг 3.7. Иначе переходим на шаг 3.2.

3.7. Сортируем ребра из $E_{Q}^{\prime}$ по убыванию весов и объявляем их непросмотренными.

3.8. Если $\left|E_{Q}^{\prime}\right|=\sum_{i=1}^{k} n_{i}-k\left(n_{i}-\right.$ количество вершин в частях $\left(G_{Q}\right)_{i}$ графа $\left.G_{Q}\left(X_{Q}, E_{Q}\right)\right)$, переходим на шаг 4 .

3.9. Возьмем очередное непросмотренное ребро $e \in E_{Q}^{\prime}$. Если таких ребер нет, переходим на шаг 4.

3.10. Исключаем ребро е из $E_{Q}^{\prime}$, если это можно сделать, не нарушив связности дерева $T_{Q}\left(X_{Q}, E_{Q}^{\prime}\right)$. Ребро е становится просмотренным. Переходим на шаг 3.8 , если исключение проводилось, и на шаг 3.9 , если нет.

4. Меняем в дереве $T_{Q}\left(X_{Q}, E_{Q}^{\prime}\right)$ все квазивершины $T_{i}$ на деревья, полученные на шаге 1. В итоге получается полное остовное дерево.

Отметим, что проверка связности кратного графа и проверка существования кратного пути между двумя вершинами выполняются с помощью полиномиальных алгоритмов, рассмотренных в статье [1]. Таким образом, каждый отдельный шаг алгоритма полиномиален. При этом на шаге 1 выполняется поиск $|Q|$ деревьев, шаги $3.2-3.6$ выполняются не более $\left|E_{Q}\right|$ раз, а шаги 3.8-3.10 - не более $\left|E_{Q}^{\prime}\right|$ раз. Остальные шаги выполняются однократно. Следовательно, алгоритм полиномиален.

Также стоит заметить, что алгоритм может быть применен и в случае, когда граф $G(X, E)$ не является делимым (шаг 3.8 в этом случае пропускается), однако тогда результирующее дерево не обязательно будет полным.

\section{Заключение}

Данная статья продолжает исследование кратных неориентированных графов, начатое в [1]. Мы ввели понятие кратного дерева и поставили задачи о минимальном и минимальном полном остовном дереве в кратном графе. Была обоснована оценка количества ребер в кратном делимом дереве и доказан критерий полноты остовного дерева в делимом кратном графе. 
Кроме того, был предложен эвристический алгоритм поиска минимального полного остовного дерева в делимом графе. Этот алгоритм обобщает известный алгоритм Краскала и является полиномиальным.

\section{Список литературы / References}

[1] Смирнов А. В., "Задача о кратчайшем пути в кратном графе”, Моделирование и анализ информационных систем, 24:6 (2017), 466-478; [Smirnov A. V., "The Shortest Path Problem for a Multiple Graph", Modeling and Analysis of Information Systems, 24:6 (2017), 788-801, (in Russian).]

[2] Cormen T. H., Leiserson C. E., Rivest R. L., Stein C., Introduction to Algorithms, 3rd ed., The MIT Press, McGraw-Hill Book Company, 2009.

[3] Berge C., Graphs and Hypergraphs, North-Holland Publishing Company, 1973.

[4] Basu A., Blanning R. W., "Metagraphs in workflow support systems", Decision Support Systems, 25:3 (1999), 199-208.

[5] Basu A., Blanning R.W., Metagraphs and Their Applications, Integrated Series in Information Systems, 15, Springer US, 2007.

[6] Рублев В. С., Смирнов А.В., "Потоки в кратных сетях", Ярославский педагогический вестник, 3:2 (2011), 60-68; [Rublev V.S., Smirnov A. V., "Flows in Multiple Networks", Yaroslavsky Pedagogichesky Vestnik, 3:2 (2011), 60-68, (in Russian).]

[7] Smirnov A. V., "The Problem of Finding the Maximum Multiple Flow in the Divisible Network and its Special Cases", Automatic Control and Computer Sciences, 50:7 (2016), 527-535.

[8] Ford L. R., Fulkerson D. R., Flows in Networks, Princeton University Press, 1962.

[9] Рублев В.С., Смирнов А.В., “Задача целочисленного сбалансирования трехмерной матрицы и алгоритмы ее решения", Моделирование и анализ информационных сиcmeм, 17:2 (2010), 72-98; [Roublev V. S., Smirnov A. V., "The Problem of Integer-Valued Balancing of a Three-Dimensional Matrix and Algorithms of Its Solution", Modeling and Analysis of Information Systems, 17:2 (2010), 72-98, (in Russian).]

[10] Smirnov A. V., "Network Model for the Problem of Integer Balancing of a Four-Dimensional Matrix", Automatic Control and Computer Sciences, 51:7 (2017), 558-566.

[11] Kruskal J. B., "On the Shortest Spanning Subtree of a Graph and the Traveling Salesman Problem", Proceedings of the American Mathematical Society, 7:1 (1956), 48-50.

Smirnov A. V., "The Spanning Tree of a Divisible Multiple Graph", Modeling and Analysis of Information Systems, 25:4 (2018), 388-401.

DOI: $10.18255 / 1818-1015-2018-4-388-401$

Abstract. In this paper, we study undirected multiple graphs of any natural multiplicity $k>1$. There are edges of three types: ordinary edges, multiple edges and multi-edges. Each edge of the last two types is a union of $k$ linked edges, which connect 2 or $k+1$ vertices, correspondingly. The linked edges should be used simultaneously. If a vertex is incident to a multiple edge, it can be also incident to other multiple edges, and it can be the common ending vertex to $k$ linked edges of a multi-edge. If a vertex is the common end of some multi-edge, it cannot be the common end of any other multi-edge. Special attention is paid to the class of divisible multiple graphs. The main peculiarity of them is a possibility to divide the graph into $k$ parts, which are adjusted on the linked edges and which have no common edges. Each part is an ordinary graph. The definition of a multiple tree is stated and the basic properties of such trees are studied. Unlike ordinary trees, the number of edges in a multiple tree is not fixed. In the article, the evaluation of the minimum and maximum number of edges in the 
divisible tree is stated and proved. Next, the definitions of the spanning tree and the complete spanning tree of a multiple graph are given. The criterion of completeness of the spanning tree is proved for divisible graphs. It is also proved that a complete spanning tree exists in any divisible graph. If the multiple graph is weighted, the minimum spanning tree problem and the minimum complete spanning tree problem can be set. In the article, we suggest a heuristic algorithm for the minimum complete spanning tree problem for a divisible graph.

Keywords: multiple graph, multiple tree, divisible graph, spanning tree, complete spanning tree, minimum spanning tree

\section{On the authors:}

Alexander V. Smirnov, orcid.org/0000-0002-0980-2507, PhD, Associate Professor

P.G. Demidov Yaroslavl State University,

14 Sovetskaya str., Yaroslavl, 150003, Russia, e-mail: alexander_sm@mail.ru

Acknowledgments:

This work was supported by the Russian Foundation for Basic Research under the Grant No 17-07-00823 A. 